\section{A confirmation that relational organization facilitates memory*}

\author{
SAMUEL A. BOBROW and RANDOLPH D. EASTON \\ University of Washington, Seattle, Wash. 98105
}

Epstein, Rock, \& Zuckerman (1960) demonstrated that Ss remembered picture paired associates presented in a relational scene better than pictures presented side by side. Their interpretation of the result was that relational organization facilitated memory. A confounding variable, however, was physical distance. That is, the pictures were physically farther apart in the side-by-side condition than in the relational condition. The present experiment independently manipulates the relational and distance variables. The results support the Epstein et al interpretation.

Epstein, Rock, \& Zuckerman (1960) demonstrated superior recall of picture paired associates for Ss who had studied a relational as opposed to a separated version of the two pictures. That is, the Ss who studied a picture of a bottle on top of a wall had higher recall scores than Ss who saw the separate pictures of a bottle and a wall. The authors concluded that relational organization facilitated the association between the picture stimuli, supporting Kohler's (1947) view that an organizational entity was the prime factor in association formation. A confounding variable, however, in the Epstein et al experiment, is physical distance, that is, the bottle and wall drawings were touching in the relational picture, while they were relatively far apart in the separated condition. Thus, it is possible that the factor which caused the recall difference between the two conditions was the distance variable, not the relational variable. The present experiment was designed to manipulate the relational and distance variables independently. METHOD

Each S studied a list of 30 picture pairs and was then tested by being presented one of thepictures as a cue for verbal recall of the name of the other picture of that pair. The relation variable was manipulated by drawing one of the items either in a stationary pose or in an action pose. Figure 1 illustrates how a hatchet could be presented either in motion (action condition) or not in motion (stationary condition). The distance variable was manipulated orthogonally by drawing the two objects of each pair either adjacent (together condition) or apart (separated condition) (see Fig. 1). Thus, there were four different presentation conditions: action-separated, action-together, stationary-separated,

*This research was supported by a Graduate School Research Fund awarded to the senior author. respectively. orthogonally to the other two, was the nature of the test stimulus. For each $\mathrm{S}$, half of the items were tested by presentation of the action version of the test stimulus and the remaining items were tested by presentation of the stationary version of the test stimulus. Specific pair and test conditions were counterbalanced across Ss. The experimental design was then a 2 by 2 by 2 factorial, with repeated measures on the test factor.

The Ss participated in groups of nine. The eight groups were assigned randomly to conditions. Pictures were presented by a Kodak Carousel Model 800 projector. The presentation time for each slide was 3 sec at study and $8 \mathrm{sec}$ at recall. The Ss were informed that some of the test stimuli would be modified in appearance. The 72 Ss were University of Washington undergraduates whose participation fulfilled an introductory psychology course requirement.

The mean numbers of correct

A third variable, manipulated

\section{Study Condition:}

Stationary - Separate

Stationary - Together
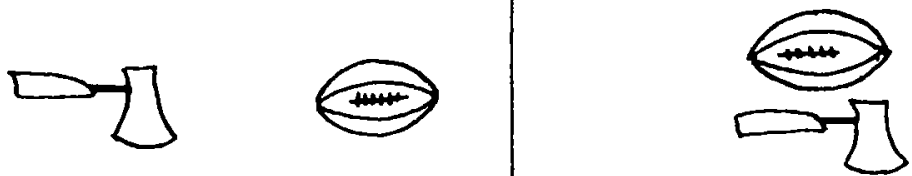

Action - Together

Action - Separate
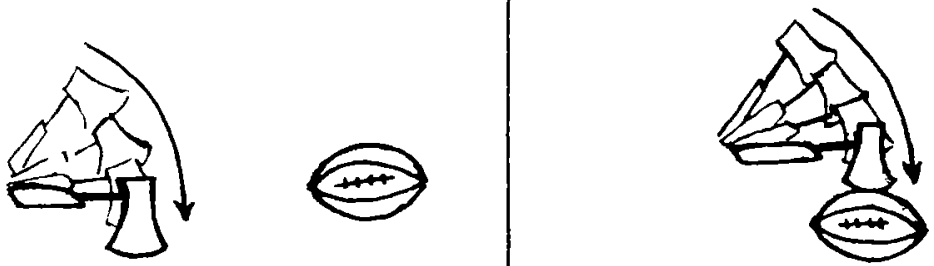

Test Cues:

Stationary

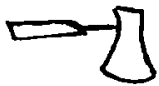

Action

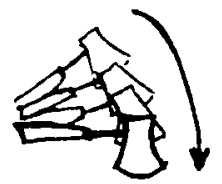

Fig. 1. Illustrations of experimental stimuli: see text for explanation. 
Table 1

Mean Correct Recall (Out of 15) Test Conditions

Stationary Action $\bar{x}$

\begin{tabular}{lccccc}
\hline \multirow{4}{*}{ Study } & $\begin{array}{c}\text { Stationary } \\
\text { Separate }\end{array}$ & 5.2 & 5.4 & 5.3 \\
& $\begin{array}{c}\text { Together } \\
\text { Action }\end{array}$ & & & & \\
Conditions & Separate & 3.2 & 3.6 & 3.4 \\
& Together & 8.7 & 8.4 & 8.5 \\
& $\mathrm{X}$ & 5.7 & 5.5 & \\
\hline
\end{tabular}

MSE (betueen $)=13.3$, MSE $($ within) $=2.3$

responses at recall for each of the eight conditions are presented in Table 1. An analysis of variance showed a significant effect due to the distance variable $\quad[F(1,68)=15.2, \quad p<.01]$. The Distance by Relational interaction was also significant $[F(1,68)=18.5$, $p<.01]$. The relational main effect was not significant, nor was the test condition variable, e.g., a stationary or action cue produced equivalent recall. The data were then combined across the nonsignificant test factor, producing a 2 by 2 factorial design with just the presentation variables of action vs stationary and separate vs together as the factors for analysis. A Newman-Keuls test was performed on the four cell totals. This test indicated that the action-together condition resulted in highest recall, followed by the two stationary conditions which were not significantly different from each other but which both yielded greater recall than the action-separate condition.

\section{DISCUSSION}

These results support the Epstein, Rock, and Zuckerman conclusion that relational organization facilitates association formation. The present experiment thus replicates recent work by Rohwer and his associates (Rohwer, Lynch, Suzuki, \& Levin. 1967 ) in showing the facilitative effects on recall of presenting interactive scenes. Presentation of two pictures close together in an action pose results in higher recall than presentation of the two pictures separated. The present experiment indicates that the closeness of the two pictures is an important variable in terms of facilitating or hindering the perception of a relationship between the stimuli. Thus, separating an action pose apparently made it difficult for Ss to perceive the relationship in the brief presentation interval. Also, presenting two nonaction pictures close together results in equivalent recall to presenting two nonaction pictures in separated form. This supports the conclusion of Asch, Ceraso, \& Heimer (1962) that perceptual conditions can influence association formation.

The fact that recall in the action-separate condition was below recall in the stationary-separate condition is difficult to account for, especially in light of the finding of no difference between presenting the action or stationary version of a picture at the time of testing. Conceivably, an action-separate input involves an additional memory load when compared with a stationary-separate input. In verbal terms, the stationary-separate condition might be represented as the hatchet and the ball, while the action-separate condition would translate as the chopping hatchet and the ball. With only 3 sec allowed for study, action-separate Ss would be spending an appreciable percentage of their time linking "chopping" and "hatchet" while stationary-separate Ss could devote the full time to linking "hatchet" and "ball." At the time of recall, $8 \mathrm{sec}$ per item was allowed, perhaps allowing Ss time to generate the stimulus image they had actually been presented for study. Systematic manipulations of study and test time intervals would yield interesting data in regard to these speculations.

The present results thus support the Epstein et al conclusion that relational organization facilitates association providing the material is presented in a manner such that thylearner can perceive a relationship.

\section{REFERENCES}

ASCH, S., CERASO, J., \& HEIMER, W. Perceptual conditions of association. Psychological Monographs, 1960, 74(3, Whole No. 490).

EPSTEIN, W., ROCK, I., \& ZUCKERMAN, C. B. Meaning and familiarity in associative learning. Psychological Monographs, $1960,74(4$, whole No. 491).

KOHLER, W. Gestalt psychology. New York: Liveright, 1947.

ROHWER, W. D., JR., LYNCH, S. SUZUKI, N., \& LEVIN, J, Verbai and pictorial facilitation of paired-associated learning. Journal of Experimental Child Psychology, 1967, 5, 294-302. 